\title{
MACTeaching: Uma Abordagem para Enriquecer o Ensino do Método de Avaliação de Comunicabilidade
}

\author{
Wagner Queiróz Junior'1, Martha Silva Fernandes², Ruddá Silva Beltrão Brito ${ }^{1}$, \\ Bruno A. Bonifácio ${ }^{1}$, Priscila Silva Fernandes ${ }^{1}$, \\ ${ }^{1}$ Instituto de Ciências Exatas e Tecnologia (ICET) \\ Universidade Federal do Amazonas (UFAM) \\ Caixa Postal 15.064 - 91.501-970 - Itacoatiara - AM - Brazil \\ ${ }^{2}$ Grupo de Usabilidade e Engenharia de Software (USES) \\ Universidade Federal do Amazonas (UFAM) \\ \{wagner.mps1, marthafernandes, beltrao.rudda, brunnoboni, \\ pry.bila\} @gmail.com
}

\begin{abstract}
The increasing evolution of computacional systems has create new challenges to develop applications that meet the expectations of users to the courses of Information Technology (IT). In this context, the Human-Computer Interaction area (HCI) brings an innovative vision of the application creation process. However teach HCI is no easy task. This paper presents the MACteaching, a game that aims to complement the teaching of communicability evaluation method, a HCI method, in a playful manner. Besides presenting the game, this paper describes its development process and its empirical evaluation, improvements to assist HCI courses.
\end{abstract}

Resumo. Com a crescente evolução dos sistemas computacionais criar novas tecnologias de software, que atendam as expectativas dos usuários traz novos desafios para os cursos de Tecnologia da Informação (TI). Nesse contexto, a área Interação Humano-Computador (IHC) traz uma visão inovadora sobre o processo de criação de aplicativos. Todavia ensinar IHC não é tarefa fácil. $O$ presente trabalho apresenta o MACteaching, um jogo que visa auxiliar o ensino do Método de Avaliação de Comunicabilidade, um método de IHC, de forma lúdica. Além de apresentar o jogo, este artigo descreve o processo de desenvolvimento e a avaliação experimental de ensino nas disciplinas de IHC.

\section{Cenário de uso}

Com o surgimento de novos paradigmas e tecnologias da informação, saber como criar novas aplicações, que atendam as expectativas dos usuários, exige conhecimento, técnicas, ferramentas e métodos que englobam tanto a Engenharia de Software como a área Interação Humano-Computador (IHC). No desenvolvimento de software, a área de IHC fornece uma visão que foca na qualidade de interação usuário-sistema, para atender de maneira satisfatória suas necessidades e expectativas dos usuários [Benitti e Sommariva 2012]. Por essa razão, o ensino de IHC tem sido cada vez mais difundido em cursos de graduação e pós-graduação na área de Tecnologia da Informação (TI), 
propiciando a formação de profissionais mais qualificados, e consequentemente, sistemas computacionais de melhor qualidade [Barbosa et al. 2010].

Assim, o uso de tecnologias de software, como recurso complementar, no processo de ensino-aprendizagem pode auxiliar na criação de atividades pedagógicas mais colaborativas e inovadoras [Brito et al. 2016]. Nesse contexto, jogos educativos podem auxiliar no desenvolvimento de habilidades cognitivas, para resolução de problemas, tomada de decisão e pensamento crítico de forma lúdica [Meireles; Bonifácio, 2015].

Diante desse cenário é que foi elaborado um jogo, para dispositivos móveis, chamado MACteaching. Essa solução utiliza uma abordagem de interação que visa melhorar a experiência de alunos no ensino de IHC, especificamente no ensino de um método fundamentado na Engenharia Semiótica, o Método de Avaliação de Comunicabilidade (MAC). A escolha do MAC é justificada devido a dificuldade que normalmente os alunos apresentam no ensino de etapas do método, o que constitui grande oportunidade de pesquisa. O MACteaching utiliza simulações para reforçar os conceitos do MAC.

O Macteaching pode ser aplicado por profissionais da área de Computação, como ferramenta complementar, para o ensino de IHC, em cursos de Engenharia de Software, Design e Sistemas de Informação. Espera-se com essa abordagem contribuir para o desenvolvimento de software, focando na qualidade de uso, no processo de construção de sistemas interativos.

\section{Desenvolvimento}

O MACteaching utiliza como abordagem interativa de aprendizagem relacional, por meio de associação por analogia. O propósito do jogo é ser uma ferramenta complementar ao ensino do método. $\mathrm{O}$ jogo caracteriza-se como um simulador prático dos conceitos do MAC, pois apresenta cenários descrevendo casos de uso que são passíveis de acontecer durante a observação do participante, na aplicação do método. Além de prática, com exercícios que favorecem o ensino das etapas do método, apresentando de maneira rápida, novas problemáticas, para que o jogador possa se familiarizar rapidamente com as etiquetas e os conceitos da área.

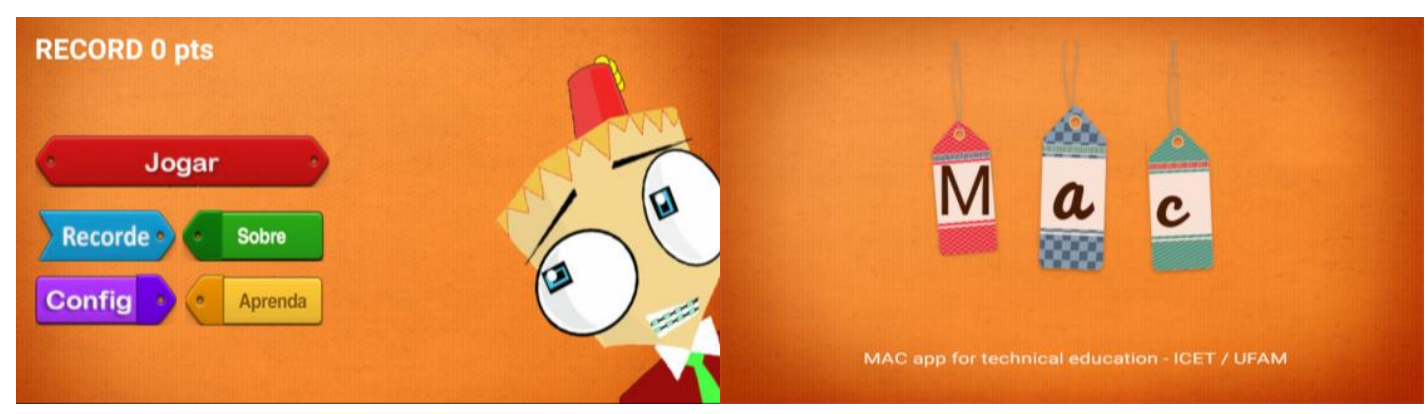

Figura 1. Interface Gráfica do Aplicativo MACTeaching 
O jogo foi desenvolvido para plataforma Android. A plataforma de desenvolvimento utilizada foi o Android Studio ${ }^{1}$ utilizando a linguagem Java, para criação e edição de imagens foi usado o Photoshop CS ${ }^{2}$.

O aplicativo encontra-se na segunda versão. Para aprimorar o aplicativo foram elaborados dois estudos, descritos detalhadamente [Brito et al. 2016], por isso será apresentado somente um resumo dos resultados do mesmo. A partir dos resultados desses estudos foi possível identificar melhorias na interface gráfica. E adicionalmente evoluir o aplicativo.

\subsection{Primeiro Estudo Experimental}

Participantes: Para este estudo foram selecionados quatro participantes, alunos de pósgraduação da Universidade Federal do Amazonas (UFAM). Adicionalmente o estudo contou com a colaboração de um observador, responsável pelo desenvolvimento do jogo. O objetivo do estudo foi avaliar quais as dificuldades em relação a interação dos usuários com o aplicativo.

Execução do Estudo: Após o uso do aplicativo, foram feitas entrevistas e aplicados questionários de avaliação sobre a experiência de uso e aprendizado dos participantes com o MACteaching. Os resultados foram agrupados em pontos fortes, pontos a serem melhorados e a percepção do participante ao usar o aplicativo como ferramenta de apoio ao ensino do Método de Avaliação de Comunicabilidade.

Resultados Obtidos: De acordo com as informações obtidas, considerando a facilidade de uso, os participantes afirmaram conseguir utilizar o MACteaching de forma satisfatória. Cerca de 55\% dos participantes afirmaram ser fácil de lembrar como utilizar o jogo. Além disso, $36 \%$ do alunos relataram o ganho de habilidade no uso do MACteaching, e 45\% que afirmaram que o MACTeaching é facil de utilizar para ensino de IHC. Como contribuição do estudo, foram feitas melhorias na aplicação, para execução de um novo estudo. Após o desenvolvimento foi feita uma nova avaliação.

A realização das entrevistas possibilitou avaliar os pontos fortes e passíveis de melhoria da aplicação. Entre os relatos obtidos podem-se destacar o obtido pelo usuário 1 " $A$ aplicação é uma ótima ferramenta para o aprendizado e assimilação da técnica MAC", aluno 4 "É iterativo, deixando o usuário com vontade de querer jogar" e o aluno 5: "Aprendi onde devo utilizar as etiquetas".

Outro ponto observado durante a avaliação foi, que a maioria dos alunos mencionaram a aprendizagem do MAC como ponto forte do jogo. Como o jogo exige agilidade do usuário, torna-se necessário o entendimento das etiquetas para se obter cada vez mais pontos.

No aspecto de sugestões e melhorias, a maioria dos alunos se sentiram prejudicadas pela falta de feedback da aplicação em relação as suas respostas, no jogo. Podemos destacar, a afirmação do alunos 1 que relatou: "A aplicação deveria informar ao usuário uma mensagem informando que o mesmo selecionou a resposta incorreta", pelo aluno 2 "Falta de resposta ao erro", e do aluno 4, que relatou: "Não aparecem mensagens

\footnotetext{
${ }^{1}$ google.android.com

${ }^{2}$ photoshop.org
} 
quando erramos". Além disso, foram observadas dificuldades dos participantes na interação com o aplicativo, principalmente para mover as etiquetas do método. Outro ponto mencionado foi a disputa com outros jogadores, que pode tornar o jogo mais atrativo.

\subsection{Segundo Estudo Experimental}

Seleção dos participantes: Para o segundo estudo foi aplicado no contexto da disciplina de IHC, no curso de Sistemas de Informação. O professor da disciplina foi responsável por supervisionar o estudo aplicado e acompanhar continuamente a interação dos alunos com o aplicativo. No total foi feito o acompanhamento na disciplina e foi coletado as opiniões dos participantes.

Indicadores: Para avaliação foi feita uma análise qualitativa, usando o Modelo de Aceitação de Tecnologia (TAM, do inglês Technology Acceptance Model). O TAM usa escalas de likert, como forma de quantificar os resultados [Prates, 2007].

Resultados Obtidos: Em relação a facilidade de uso, 78\% dos participantes concordaram em relação ao ganho de habilidade e interação usando aplicativo. Sobre a utilidade do aplicativo, cerca de $87 \%$ dos participantes relataram que o aplicativo pode auxiliar no ensino do MAC, e de conceitos aplicados em IHC e Engenharia de Software. Adicionalmente, em relação a técnica usada no jogo, de simulação por meio de história, a maioria dos participantes relataram satisfação com a narrativa usada no jogo.

Contribuições: Foram identificados um total de cinco pontos de melhorias como: dificuldade em saber para onde arrastar as etiquetas, a velocidade da história inicial do jogo, dificuldade de leitura em legendas do jogo; feedbacks do jogo sem destaque e; tempo de jogo muito curto.

\section{Apresentação do Software}

A história apresentada no jogo é centrada em uma cidade, chamada Interacionópolis, onde vivem desenvolvedores e designers produzindo sistemas. Porém, surge uma bruxa, chamada Ruptura, que se incomoda com o sucesso dos desenvolvedores e começa a inserir várias rupturas de interação nas interfaces dos sistemas gerados dentro da cidade. Assim o objetivo é identificar as rupturas para que o personagem principal possa vencer a bruxa.

Para resolver a situação, uma equipe de desenvolvimento resolve aplicar o Método de Avaliação de Comunicabilidade (MAC). O jogo então tem o propósito de identificar as rupturas nos sistemas, onde o jogador do aplicativo atua como personagem principal, um participante chamado Meckinho. O objetivo é identificar, para cada ruptura qual a etiqueta do método que expressa o problema de comunicação na interface gráfica dos sistemas interativos.

Para a interação realizada pelo jogador durante a simulação, foi utilizado um mecanismo de drag-and-drop. O objetivo do jogo é conseguir etiquetar o máximo de rupturas corretamente e salvar a cidade da bruxa Ruptura. $\mathrm{O}$ jogo disponibiliza ainda descrições das treze etiquetas do MAC, com interface intuitiva e amigável. 
VI Congresso Brasileiro de Informática na Educação (CBIE 2017)

Anais dos Workshops do VI Congresso Brasileiro de Informática na Educação (WCBIE 2017)
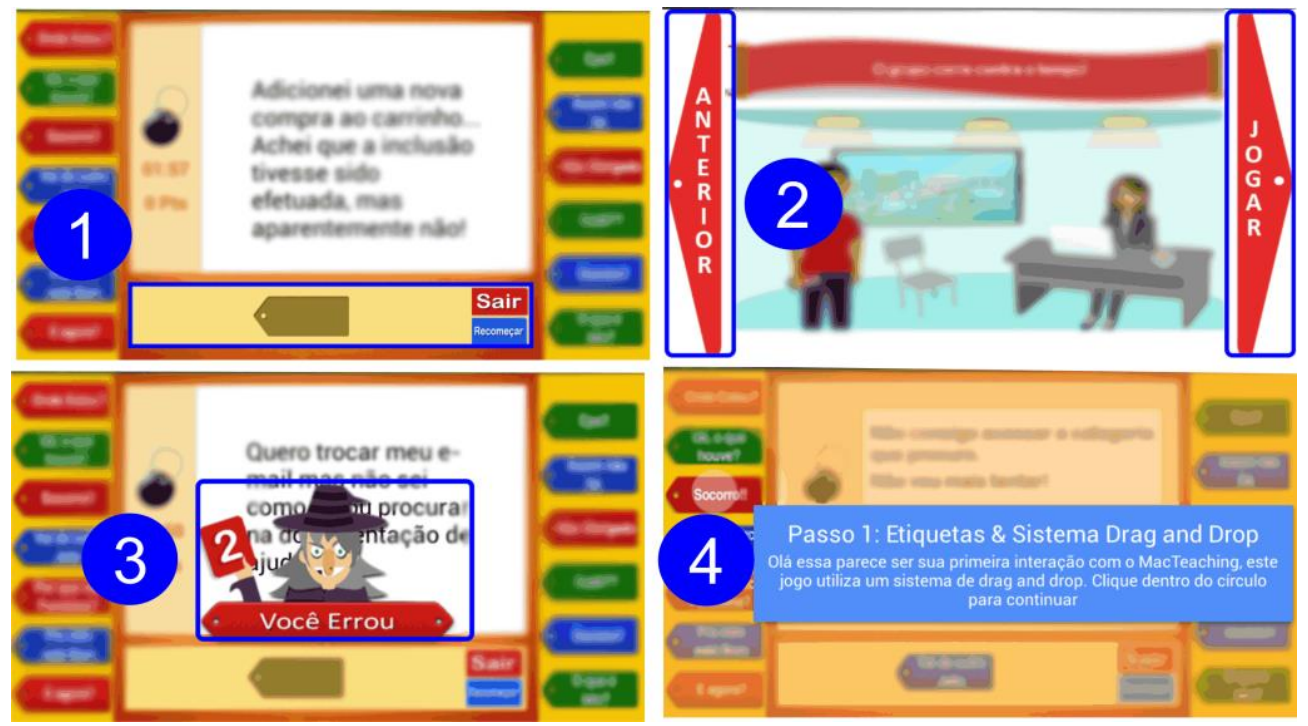

Figura 2. Interface gráfica do aplicativo após o estudo.

A Figura 5 apresenta a interface gráfica atual do aplicativo tais como: indicação na interface gráfica do aplicativo mostrando o local de movimento das etiquetas (Figura 5-1); novos botões de controle avançar/jogar e voltar para melhor leitura da historinha do jogo (Figura 5-2); feedback após interação do jogo, com imagens que ilustram a resposta do usuário (Figura 5-3); aumento do tempo após iniciar a partida, além de ranking dos 10 (dez) melhores jogadores.

Por fim, um tutorial sobre o funcionamento do jogo, mostrando instruções e pontos que auxiliam no aprendizado do jogo e do MAC (Figura 5-4). A nova versão desenvolvida do MACteaching, ainda em desenvolvimento, se encontra disponível na loja de aplicativos Play Store ${ }^{3}$. Para melhor divulgação o vídeo se encontra disponível na plataforma do Youtube ${ }^{4}$.

\section{Considerações finais}

O presente trabalho apresentou um aplicativo para o ensino de IHC, nos cursos de Computação, chamado MACTeaching. O aplicativo é uma proposta complementar ao ensino do MAC. Como característica principal é o uso de analogias para tornar o ensino mais prazeroso. O MACTeaching encontra-se na sua segunda versão, os resultados dos dois estudos indicam melhorias que podem tornar um importante instrumento de aprendizagem. As contribuições permitirão evoluir a aplicação para um sistema colaborativo, alimentado por educadores que poderá tornar o aplicativo customizável.

Como trabalho futuro pretende-se, ainda: (1) desenvolver novos requisitos e novos fluxos para melhoria do aplicativo, transformando o MACteaching em um plataforma colaborativa, para permitir que profissionais da educação utilizem o aplicativo dentro e fora da sala de aula, com a possibilidade de customização das questões utilizadas no jogo, inseridas pelos próprios pesquisadores e educadores da área

\footnotetext{
${ }^{3}$ https://play.google.com/store/apps/details?id=macapp.ufam.icet.com.mac

${ }^{4}$ https://www.youtube.com/watch?v=zwExKLULmKI
} 
VI Congresso Brasileiro de Informática na Educação (CBIE 2017)

Anais dos Workshops do VI Congresso Brasileiro de Informática na Educação (WCBIE 2017)

de IHC e Engenharia de Software; (2) e realizar uma nova avaliação para avaliar o impacto real do aplicativo no ensino de IHC, comparando com a metodologia de ensino tradicional e utilizando o aplicativo.

Espera-se com este artigo, contribuir para o processo de ensino e aprendizagem da Engenharia de Software e de IHC, buscando proporcionar melhor qualificação dos profissionais, por meio da teoria aliada a prática, utilizando tecnologias de software, proporcionando a criação de práticas pedagógicas inovadoras de ensino.

\section{Referências}

Brito, R., Queirós JR, W., Fernandes, M., Bonifácio, B. Fernandes, P., (2015) " MACteaching: Utilizando um Jogo para Apoio ao Ensino do Método de Avaliação de Comunicabilidade" In: XXVII Simpósio Brasileiro de Informática na Educação, 2016, Uberlandia, 2016. p. 180-189.

Barbosa, J.D.S. Silva, S.B. (2010). Interação Humano - Computador. 1ra ed. Elsevier, Rio de Janeiro, 2010, pag 385.

Meireles, M.C. and Bonifácio, B. A. (2015) "Uso de Métodos Ágeis e Aprendizagem Baseada em Problema no Ensino de Engenharia de Software: Um Relato de Experiência." In: XXVI Simpósio Brasileiro de Informática na Educação, 2015, Maceió, 2015. p. 180-189.

Prates, R.O and Barbosa, S.D.J .(2007) "Introdução à Teoria e Prática da Interação Humano- Computador fundamentada na Engenharia Semiótica". In T. Kowaltowski \& K. Breitman(orgs) Jornadas de atualização em Informática.

Sommariva, L. Benitti, F. B.V. and Dalcin, F.S. (2011). "UsabilityGame: jogo simulador para apoio ao ensino de usabilidade". In: Proceedings of the 10th Brazilian Symposium on on Human Factors in Computing Systems and the 5th Latin American Conference on Human-Computer Interaction. Brazilian Computer Society, 2011. p. 61-65. 